\title{
ARTICLE OPEN \\ Structural transformation of layered double hydroxides: an in situ TEM analysis
}

Christopher Hobbs ${ }^{1,2,3}$, Sonia Jaskaniec ${ }^{2,4}$, Eoin K. McCarthy ${ }^{2,3}$, Clive Downing ${ }^{2,3}$, Konrad Opelt ${ }^{5}$, Konrad Güth ${ }^{5}$, Aleksey Shmeliov ${ }^{1,3}$, Maurice C. D. Mourad ${ }^{6}$, Karl Mandel ${ }^{7,8}$ and Valeria Nicolosi ${ }^{2,4}$

A comprehensive nanoscale understanding of layered double hydroxide (LDH) thermal evolution is critical for their current and future applications as catalysts, flame retardants and oxygen evolution performers. In this report, we applied in situ transmission electron microscopy (TEM) to extensively characterise the thermal progressions of nickel-iron containing (Ni-Fe) LDH nanomaterials. The combinative approach of TEM and selected area electron diffraction (SAED) yielded both a morphological and crystallographic understanding of such processes. As the Ni-Fe LDH nanomaterials are heated in situ, an amorphization occurred at $250^{\circ} \mathrm{C}$, followed by a transition to a heterogeneous structure of $\mathrm{NiO}$ particles embedded throughout a $\mathrm{NiFe}_{2} \mathrm{O}_{4}$ matrix at $850^{\circ} \mathrm{C}$, confirmed by highresolution TEM and scanning TEM. Further electron microscopy characterisation methodologies of energy-filtered TEM were utilised to directly observe these mechanistic behaviours in real time, showing an evolution and nucleation to an array of spherical NiO nanoparticles on the platelet surfaces. The versatility of this characterisation approach was verified by the analogous behaviours of $\mathrm{Ni}$-Fe LDH materials heated ex situ as well as parallel in situ TEM and SAED comparisons to that of an akin magnesium-aluminium containing (Mg-Al) LDH structure. The in situ TEM work hereby discussed allows for a state-of-the-art understanding of the Ni-Fe material thermal evolution. This is an important first, which reveals pivotal information, especially when considering LDH applications as catalysts and flame retardants.

npj 2D Materials and Applications (2018)2:4 ; doi:10.1038/s41699-018-0048-4

\section{INTRODUCTION}

In recent years, two-dimensional (2D) nanomaterials have been described as strongholds across the fields of nanotechnology with extensive applications in electronics, catalysis, drug delivery, photonics or magnetics. ${ }^{1}$ Layered double hydroxides (LDHs) are a particular class of 2D materials. Described as a member of the anionic clay family, LDHs are composed of 'brucite-like' cationic layers where an inclusion of trivalent cations introduces an overall positive charge to the nanosheets. Charge compensating anions are located in the interlayer galleries leading to the generalised $\mathrm{LDH}$ formula, $\left[\mathrm{M}^{2+}{ }_{1-x} \mathrm{M}^{3+}{ }_{x}(\mathrm{OH})_{2}\right]^{x+}\left[\mathrm{A}^{n-}\right]_{x / n} \cdot \mathrm{mH}_{2} \mathrm{O}$, where $\mathrm{M}^{2+} / \mathrm{M}^{3}$ ${ }^{+}=$divalent/trivalent metal cation and $\mathrm{A}^{n}=$ interlayer. $^{2} \mathrm{~A}$ representation of a general LDH structure is displayed in Fig. 1.

LDHs are versatile materials with high industrial and academic interest, having applications in drug delivery, ${ }^{3}$ water oxidation, ${ }^{4}$ catalysis, ${ }^{5}$ supercapacitors, ${ }^{6}$ gas absorbents, ${ }^{7}$ nanocomposites, ${ }^{8}$ and transistors, ${ }^{9}$. Two exciting members of the $\mathrm{LDH}$ family are magnesium-aluminium (Mg-Al) LDH and nickel-iron (Ni-Fe) LDH. The former has previously been applied in flame retardant studies, $^{10,11}$ polymer composites ${ }^{12}$ and catalyst supports. ${ }^{13}$ The latter also exhibits broad applicability in flame retardancy, ${ }^{14}$ sensors, ${ }^{15}$ electrocatalysis, ${ }^{16}$ water oxidation, ${ }^{17}$ oxygen evolution ${ }^{18}$ and energy storage. ${ }^{19}$ The application of Ni-Fe LDH in these fields is largely owed to their inherent material properties such as simplistic methods of fabrication, low cost, large specific surface areas, greater active sites and ability to interact with various catalytic supports. In fact, the calcination of LDH materials in general has received an abundance of attention from both an applications and scientific perspective. ${ }^{20-22}$ The associated mechanisms of this thermal degradation have also been well established and described via a complex procedure of dehydration, dehydroxylisation and decarbonisation of the lamellar material. ${ }^{23,24}$ While factors such as composition, ${ }^{25}$ morphology, ${ }^{26}$ structure and atmospheric conditions ${ }^{27}$ can affect these calcination behaviours, many studies have utilised the calcination procedures for applications in areas such as oxygen evolution ${ }^{16}$ and catalytic supports. ${ }^{13}$ In particular, it is the generation of metal oxides and spinel structure composites derived from LDH calcinations that contribute to the enhancement of catalytic properties across several catalysis-related fields including nanocatalyst design, ${ }^{28,29}$ hydrogenation ${ }^{30}$ and photocatalysis. ${ }^{31}$ Moreover, the application of LDH materials in flame retardant composites has attracted interest in recent times. The incorporation of the lamellar structures into polymer materials has shown beneficial results of thermal stability, melting temperatures and smoke suppression as well as an attenuation of peak heat release rate in a number of cases. ${ }^{32}$ Evidently, the thermal decomposition of the LDH structures plays an important role in both catalytic performance and flame retardant properties of LDH nanocomposites. Despite this extensive work throughout such areas of

\footnotetext{
${ }^{1}$ School of Physics, Trinity College Dublin (TCD), Dublin, Ireland; ${ }^{2}$ Advanced Materials and Bioengineering Research Centre (AMBER) and Centre for Research of Adaptive Nanostructures and Nanodevices (CRANN) Trinity College Dublin (TCD), Dublin, Ireland; ${ }^{3}$ The Advanced Microscopy Laboratory, CRANN Trinity College Dublin (TCD), Dublin, Ireland; ${ }^{4}$ School of Chemistry Trinity College Dublin (TCD), Dublin, Ireland; ${ }^{5}$ Fraunhofer-Project Group Materials Recycling and Resource Strategies IWKS 63457 Hanau, Germany;

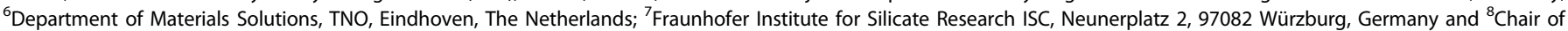
Chemical Technology of Materials Synthesis, Department Chemistry and Pharmacy, Julius-Maximilians University Würzburg, Röntgenring 11, 97070 Würzburg, Germany Correspondence: Valeria Nicolosi (nicolov@tcd.ie)
} 


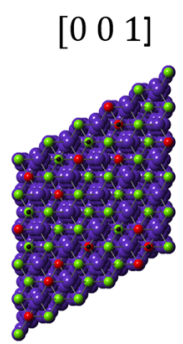

\author{
$\left[\begin{array}{lll}0 & 1 & 0\end{array}\right]$
}
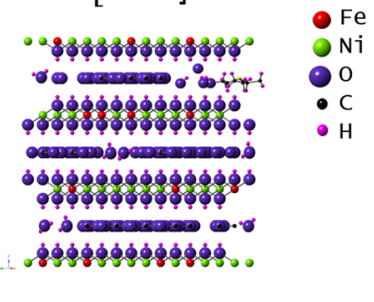

Fig. 1 Schematic representations of the as-synthesised Ni-Fe LDH structure, viewed along the [001] and [010] directions. (Structures were visualised using CrystalMaker ${ }^{\mathrm{TM}}$ software.) Cationic layers composed of $\mathrm{Ni}$ and $\mathrm{Fe}$ octahedrally surrounded by hydroxides are charge compensated by anionic interlayer moieties such as carbonate $\left(\mathrm{CO}_{2}{ }^{3-}\right)$, water $\left(\mathrm{H}_{2} \mathrm{O}\right)$ and triethanolamine $\left(\mathrm{C}_{6} \mathrm{H}_{15} \mathrm{NO}_{3}\right)$

research, the majority of these works have largely relied on macroscopic characterisation techniques such as X-ray diffraction (XRD), Fourier transform infrared spectroscopy (FTIR) and thermogravimetric analysis (TGA). ${ }^{8,27,33-35}$ In addition to these characterisation techniques, pre-mortem and post-mortem electron microscopy can provide important information. However, none of these pre-mortem and post-mortem methods can be used to elucidate on how to optimise the processes that occur under realtime conditions at single nanoparticle level. A detailed in situ TEM characterisation, observing thermal transformations at individual nanoparticle level, has yet to be established. Determining the correct structure-property-function relationships requires a detailed description of the material in its working state. Recent technological advances in EM allow us to image materials with a range of in situ techniques that have been developed to follow the evolution of materials in the presence of such external stimuli. In addition to observing materials in their working state, in situ techniques can also capture important intermediate transitional forms that may be involved in phase transformations. As such, previous research acknowledges that these exact transformations in which the LDH decomposes are not fully understood. ${ }^{10}$ Utilising such in situ techniques help to answer the underlying questions regarding the thermal transformations of the $\mathrm{LDH}$ material from a nanoscale perspective. Ultimately, these behaviours can be monitored and analysed in real time, providing a further understanding and optimisation of the related processes. Here, we report on the application of state-of-the-art in situ transmission electron microscopy to reveal the processes by which LDHs thermally decompose and to characterise the stages at which these morphological and crystallographic alterations occur. Due to their broad application and well-established calcination processes in the previous literature, we elected to focus our attention on MgAl LDH and Ni-Fe LDH for in situ TEM analysis. Undoubtedly, there is an urgent need to fully characterise and understand these materials from a single particle perspective, given their prospective nanoscale applications in flame retardancy, energy storage and catalysis.

\section{RESULTS AND DISCUSSION}

In both Mg-Al LDH and Ni-Fe LDH cases, a molar ratio of the cationic sites of 3 was selected as a representative of these LDH compositions due to its well-established behaviours and previous successful applications. ${ }^{36-40}$ The as-synthesised materials were first characterised by XRD, FTIR, TGA and atomic absorption spectroscopy (AAS). The FTIR spectrum of the precursor Ni-Fe and Mg-Al LDH samples is shown in Figure S1a. The broad bands at approximately 3,400 and $1,645 \mathrm{~cm}^{-1}$ are due to the various types of $\mathrm{O}-\mathrm{H}$ bonding in both materials such as the hydroxyl groups on the brucite-like layers and the adsorbed/inter-gallery water molecules. The sharp absorption band at approximately $1350 \mathrm{~cm}^{-1}$ is ascribed to the stretching modes of $\mathrm{CO}_{3}{ }^{2-}$, originating from the intercalated carbonates. ${ }^{39}$ There is also evidence of $\mathrm{C}-\mathrm{N}$ stretching modes coming from tertiary amines at approximately 1,155 and $1,040 \mathrm{~cm}^{-1}$ absorption bands in the FTIR spectrum. Moreover, absorption bands below $1,000 \mathrm{~cm}^{-1}$ are attributed to lattice stretching modes of metal-O sites. TGA analysis was also conducted on both LDH compositions (Figure $\mathrm{S} 1 \mathrm{~b})$. Firstly, there is a significant mass loss observed (10\%) in the $75-150^{\circ} \mathrm{C}$ range and is attributed to the dehydration of surface adsorbed and interstitial water. Further mass losses (approximately $20 \%$ ) in the $200-800{ }^{\circ} \mathrm{C}$ temperature range is also observed for both compositions, and is related to the dehydroxylation and decomposition of the counter ions and brucite-like layers themselves. ${ }^{41}$ AAS analysis demonstrated a Ni:Fe molar ratio of 3.54:1.

In situ and ex situ heating TEM experiments were comparatively carried out in parallel to fully understand the development of LDH thermally induced calcination, as well as the various crystallographic transitions occurring before that. For this purpose, the as-synthesised LDH samples were subjected to identical heating ramp conditions both in ex situ and in situ experimental set-ups (Figure S2). Further details can be found in the Methods section.

This parallel set-up determined if the behaviours of LDH transformations were independent of the different in situ and ex situ experimental conditions. For example, the significant pressure variations as the samples were heated may have impacted the thermal behaviours of the LDH nanomaterials. More importantly, there can be an appreciable contribution of radiation beam damage (in the form of radiolysis or knock-on) or Joule sample heating when the electron beam interacts with the sample. ${ }^{42}$ In view of these possible issues, full TEM characterisation of the various LDH samples were performed pre and post ex situ heating experiments, as well as during in situ analysis; this comparison was of crucial importance in order to rule out any eventual electron beam-induced transition.

We investigated if the LDH degradation processes are dependent on both the composition and platelet size of the material. As such, the structural transformation of a Ni-Fe LDH material was studied via the application of in situ heating TEM. Fig. 2 displays the BFTEM and associated SAED patterns at various stages of the in situ heating experiments for the Ni-Fe LDH material. Initially, the material was synthesised to have a welldefined hexagonal morphology with a lateral dimension on the order of microns. This starting material was found to have an LDH crystallographic structure, with the associated SAED pattern revealing the (101), $(01 \overline{1}),(11 \overline{2})$ and (110) crystallographic planes (Fig. 2e). Upon heating, both a morphological and crystallographic change was evidenced. As the sample reached $250^{\circ} \mathrm{C}$, the loss of the (101), (01 $\overline{1})$ and $(11 \overline{2})$ reflections indicates a collapse in the crystal structure and a reduction in the non-basal crystallographic phases while still retaining its hexagonal morphology. The loss of such planes is believed to be due to the dehydration of the interstitial galleries. Moreover, the contrast variations of BFTEM images (Fig. 2a, b) indicates a surface alteration at this temperature, possibly as a consequence of the additional loss of water both from the surface of the material and the interlayer galleries. The irradiation of the electron beam perhaps also contributed to the dehydration of the LDH materials; however, this is difficult to avoid. ${ }^{43}$ As the Ni-Fe LDH was heated to $450^{\circ} \mathrm{C}$, the well-defined hexagonal morphology is retained (Fig. 2c). However, the evolution of spherical particles, $<50 \mathrm{~nm}$ in size, were seen to generate and randomly distribute themselves onto the hexagonal parent material (Fig. 2c). There appears to be minimal crystallographic tranformations from $250^{\circ} \mathrm{C}$ to $450^{\circ} \mathrm{C}$ (Fig. 2f, g). This is indicated by the conservation of the (110) LDH plane. As the sample was calcined to $850{ }^{\circ} \mathrm{C}$, there is clear evidence of a further generation of these particles (Fig. $2 \mathrm{~d}$ ). These nucleations become 

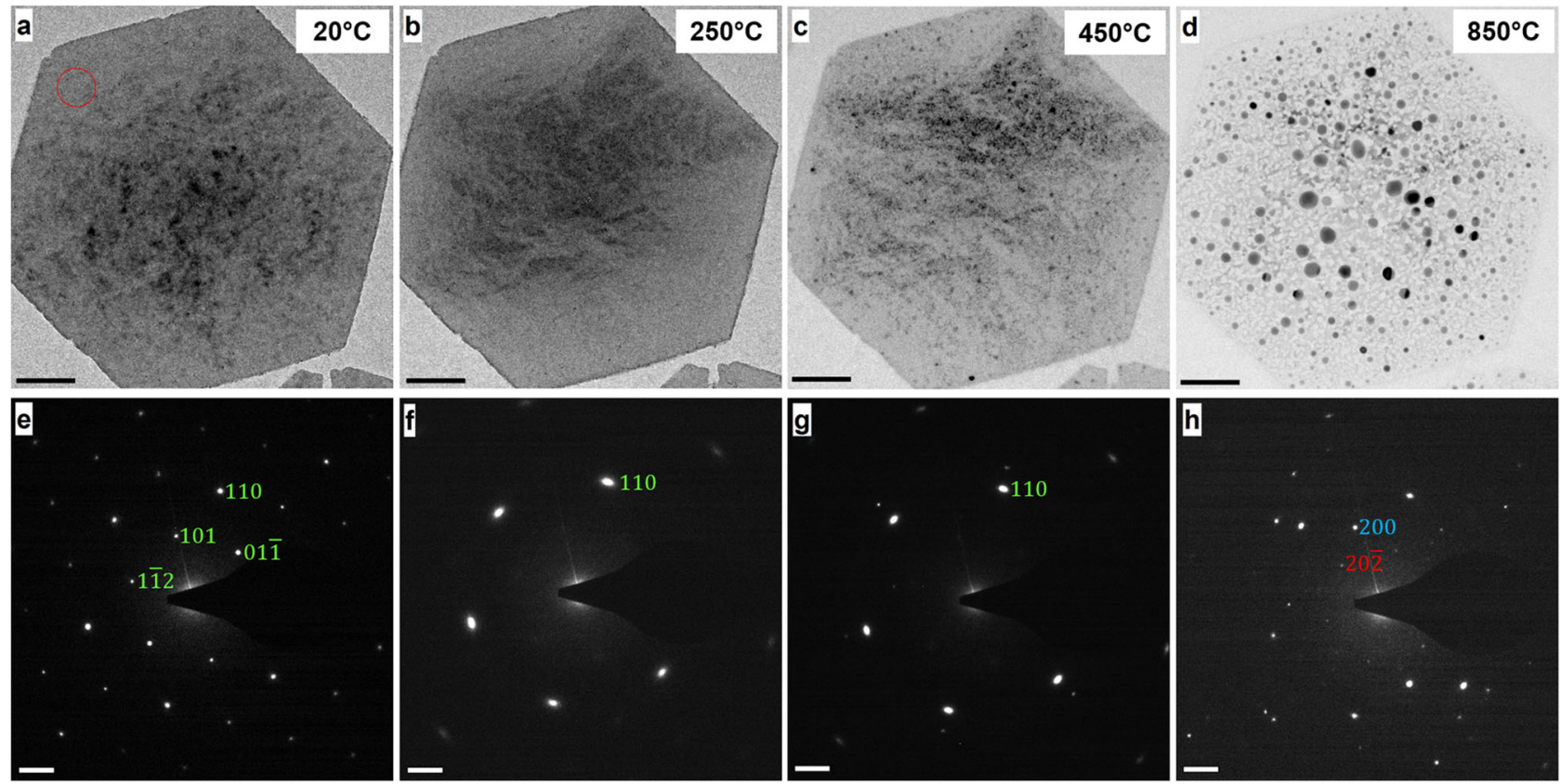

Fig. 2 BFTEM and associated SAED patterns from various stages of the in situ heating experiments of the Ni-Fe LDH. a-d BFTEM and e-h SAED patterns corresponding to column temperature, end of the $250^{\circ} \mathrm{C}$ step, end of the $450{ }^{\circ} \mathrm{C}$ step and end of the $850^{\circ} \mathrm{C}$ step, respectively. Scale bars are a-d $200 \mathrm{~nm}$ and $\mathbf{e}-\mathbf{h} 2 \mathrm{~nm}^{-1}$, respectively

more numerous, increase in size and randomly distribute themselves across the hexagonal LDH platelet (Fig. 2d). The SAED pattern of the calcined sample at $850^{\circ} \mathrm{C}$ shows a crystal structure alteration (Fig. $2 \mathrm{~h}$ ). In contrast to the patterns recorded at $250^{\circ} \mathrm{C}$ and $450^{\circ} \mathrm{C}$, there is a generation of new reflections in the diffraction pattern, corresponding to newly arranged crystallographic planes. The evolution of such particles and a variance in the diffraction patterns during the calcination procedures confirms a rearrangement of the $\mathrm{LDH}$ structures into a new crystallographic phase. It is believed that this is a topotactic transformation caused by the in situ heating effects. Further analysis of the SAED patterns recorded at $850^{\circ} \mathrm{C}$ from in situ TEM experiments (Fig. $2 \mathrm{~h}$ ) portrays the emergence of the $\{200\}$ and $\{220\}$ family of planes of the $\mathrm{NiO}$ and trevorite structures, respectively. The reflections occurring at $13.1 \mathrm{~nm}^{-1}$ may also derive from the parent LDH structure. This suggests that certain $\mathrm{LDH}$ planes may be retained even after thermal degradation procedures. In this regard, the SAED findings revealed an evolution to a mixed phase of $\mathrm{LDH}$, metal oxides and trevorite structures (Fig. 2e-h).

To further understand the characteristics of the calcined $\mathrm{Ni}-\mathrm{Fe}$ structures, we post-analysed the Ni-Fe LDH samples using energyfiltered TEM (EFTEM) and high-resolution TEM (HRTEM) (Figs. 3 and 4 , respectively). Further details of the EFTEM technique can be found in the Methods section. EFTEM was favoured over scanning TEM (STEM) for acquiring spectroscopic data in situ such as energy-dispersive $\mathrm{x}$-ray (EDX) and electron energy loss spectroscopy (EELS) to reduce the risk of inducing further transformations due to the beam-sample interaction and not as a consequence of applied thermal environments. Also, the application of EFTEM allowed for the direct analysis of the $\mathrm{Ni}$ and Fe from the whole platelet via the acquisition of two EFTEM maps (Fig. 3a). This application was preferred over STEM-EELS methods as a longer timescale for spectroscopic acquistions is required for such LDH platelet regions. The STEM approach may have also introduced issues of drift and potential alterations of crystallographic environments and, in turn, would have impacted the true reflection of the $\mathrm{Ni}$-Fe $\mathrm{LDH}$ calcination behaviours.

EFTEM revealed the distribution of $\mathrm{Ni}$ and $\mathrm{Fe}$ throughout the calcined products (Fig. 3a). In contrast to the Ni evolution, Fe was found to have a more regular distribution across the platelet, with a localisation in the centre of the calcined material. Larger $\mathrm{NiO}$ particles tended to form on the central sections of the platelets, with smaller $\mathrm{NiO}$ crystallites arranged on the platelets edge regions. This may have been due to the non-uniform local crystallographic environments of the cationic sites, whereby the different environments at the edge regions of the platelets affected the growth of both $\mathrm{NiFe}_{2} \mathrm{O}_{4}$ trevorite and $\mathrm{NiO}$ oxide nanoparticles. Moreover, it is perceived that the catalytic activity at these edge regions were not only influenced by Fe sites ${ }^{44}$ but were also influenced by smaller sized $\mathrm{NiO}$ particles. We also used the EFTEM technique to directly visualise the $\mathrm{Ni}$-Fe thermal transformations as they took place (Movie V1 Supplementary Information). Our experimental set-up utilised signals from the excitations of the electrons in the $\mathrm{Ni} 2 p_{3 / 2}$ orbital $(855 \mathrm{eV})$, that is, the $\mathrm{Ni} \mathrm{L}_{3}$ EELS edge, to form the intensities in such video representations. ${ }^{45} \mathrm{As}$ the platelets were subject to $850^{\circ} \mathrm{C}$, the nucleation of $\mathrm{Ni}$ containing particles was clearly evidenced. Particles initially evolved and migrated in a random fashion on the platelet surfaces. Further exposure at this temperature led to a sudden transformation where the remaining material transformed to an array of smaller similar $\mathrm{Ni}$ containing particles. This somewhat restricted size is perhaps due to the limited local availability of Ni cations after the initial transformations occurred.

Complimentary to the EFTEM data, an elemental distribution of the calcined Ni-Fe LDH material was further analysed in STEM. An associated EDX elemental map confirmed that the spherical nanoparticles were largely composed of nickel (green signal) (Figure S6B). The effect of the background was corrected for by the subtraction of the average intensity value in the related $\mathrm{Fe}$ map (red signal) (Figure S6B). It is also noted that small amounts of Fe may have been present either within or in the vicinity of the particle, suggested by the feint red intensities across the 

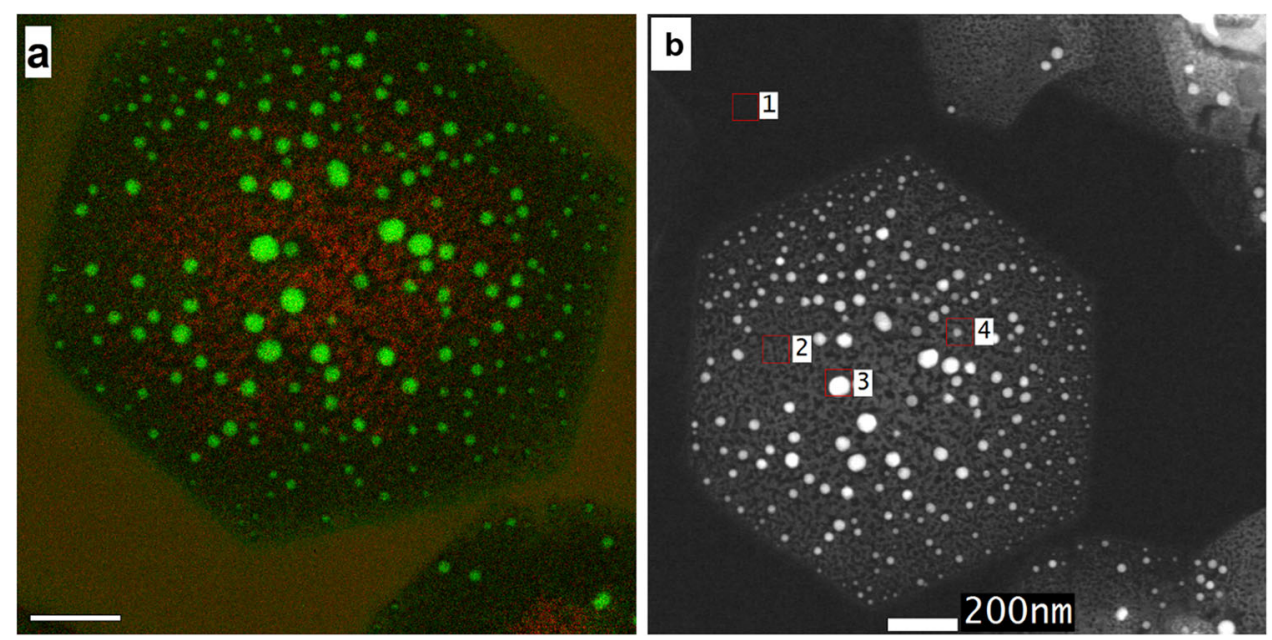

Fig. 3 a Energy-filtered TEM image of Ni-Fe LDH sample post-heating. Ni and Fe signals are represented by the colours green and red, respectively. $\mathbf{b}$ Scanning transmission electron microscopy image of the same platelet. Areas 1-4 indicated in $\mathbf{b}$ represent regions from which EDX spectra were acquired. Scale bar in a is $200 \mathrm{~nm}$

elemental map. Moreover, the low amounts of Fe was illustrated by the recorded EDX sum spectra from areas 1 to 3 across the particle (Figure S6C and D). Although this may have suggested that there is an existence of Fe migration into the $\mathrm{NiO}$ particles, the Fe-K EDX line peak at $6.4 \mathrm{keV}$ was not statistically significant across the particle and was deemed to be undetectable above background counts. Hence, the Fe signals are speculated to be due to EDX background counts.

Furthermore, we employed electron diffraction in STEM mode to investigate the variation in the orientation of the metal oxides (Supporting Information Figure S3). This overcame the drawback of SAED techniques in TEM, where the dimensions of the selected area apertures limit the recording of diffraction patterns from regions of minimum $100 \mathrm{~nm}$ in size. Whereas the application of electron diffraction using a STEM electron probe permitted the characterisation of features of approximately $10 \mathrm{~nm}$, that is, the size range of the products of calcination procedures. In addition, the application of electron diffraction studies in STEM allowed us to investigate the crystallographic orientations across the calcined $\mathrm{Ni}$-Fe structures. The variations in the recorded patterns show that the $\mathrm{NiO}$ particles assembled with random orientations relative to the basal plane of the LDH platelet (Figure S3d and f). This is also viewed in the $\mathrm{NiFe}_{2} \mathrm{O}_{4}$ trevorite regions of the calcined structure (Figure $\mathrm{S} 3 \mathrm{C}$ and e). It is deduced that the products of mixed metal oxides derived from the $\mathrm{Ni}-\mathrm{Fe} \mathrm{LDH}$ calcinations arrange themselves randomly in a topotactic fashion throughout the parent material, but confine themselves to within the platelet domains.

Energy-dispersive $x$-ray spectroscopy in STEM quantitatively assessed the calcined products of the Ni-Fe degradations. While the Fe content appeared to be ubiquitously distributed, STEM-EDX revealed that there is a notable higher Ni content on the thermally evolved spherical particles (Fig. $3 \mathrm{~b}$ and Table 1 (Supplementary Information)). This yielded additional evidence that the evolved particles are that of $\mathrm{NiO}$, and also correlated well with the data acquired by EFTEM methods.

An investigation by HRTEM showed the presence of the $\mathrm{NiFe}_{2} \mathrm{O}_{4}$ trevorite lattice structure surrounding the spherical particles resulting in a heterogeneous structure (Fig. 4e). FFT analysis of the associated regions (Fig. $4 \mathrm{~g}$ ) confirmed the existence of the $\{220\}$ and $\{242\}$ family of planes (indicated by red indices in Fig. $4 \mathrm{~g}$ ), where the crystallite region had a $[\overline{1} 1 \overline{1}]$ orientation. This can also be corroborated to the Fe signal as found by EFTEM methods (Fig. 3a). Similar analysis of the spherical nanoparticles directly resolved the lattice spacings attributed to the $\{200\}$ planes of the $\mathrm{NiO}$ structure with an orientation along the [023] axis, hence confirming the evolution of metal oxide particles as a result of the $\mathrm{Ni}$-Fe LDH calcination. However, there was a discrepancy in the correlation of the STEM nanoprobe diffraction and HRTEM of similar particle regions, whereby the $(11 \overline{1})$ family of planes found in the STEM nanoprobe acquisitions did not fully coincide with the FFT analysis of our HRTEM data (Fig. 4c) and Supporting Information S3f. We perceive this to be due to the evolution of a non-stoichiometric $\mathrm{NiO}$ compound as opposed to a pure $\mathrm{NiO}$ phase. The existence of non-stochiometric NiOs has been previously investigated by Da Rocha and Rougier. ${ }^{46}$

As aforementioned, the parallel TEM analysis of samples heated ex situ investigated the applicability of our in situ EM methodologies as well as assessed the effect of the electron beam on the $\mathrm{LDH}$ thermal evolution. Figure 5 presents the TEM and SAED studies applied to Ni-Fe LDH samples heated with ex situ experimental conditions.

The samples heated to $250^{\circ} \mathrm{C}$ ex situ presented an irregular platelet morphology (Fig. 5a). This evidently contrasts the precursor LDH platelets as well as the LDH materials heated to $250^{\circ} \mathrm{C}$ using in situ methods (Fig. 2a, b, respectively). The corresponding SAED pattern showed that these irregular sheetlike materials display an LDH crystallographic structure, demonstrated by the $\{101\}$ and $\{110\}$ planes (Fig. 5d). Similarly, these crystallographic planes were evidenced as the sample was heated to $450^{\circ} \mathrm{C}$ using ex situ methods (Fig. 5e). The BFTEM findings (Fig. $5 b)$ are also consistent with in situ studies at this temperature, in particular the irregular morphology and formation of particles on the platelets surface (Fig. 5b). The morphology demonstrated an arrangement of smaller crystallite regions at $850^{\circ} \mathrm{C}$ (Fig. 5C) with similar crystallographic features (Fig. 5f). These observations of ex situ heated materials may also be perceived as a reconstructed $\mathrm{LDH}$ due to its exposure to aqueous environments, that is, the LDH 'memory effect'. ${ }^{7}$ Moreover, the effect of rehydration due to exposure to air conditions can also be viewed from the analysis of the in situ samples, 40 days after experimentation (Figure S7). The hexagonal symmetry in the associated SAED patterns highlighted a partial revert to the LDH structure (Fig. $5 f$ and Figure S7B). However, the existence of trevorite phases remain, as shown by HRTEM analysis in both ex situ and in situ experiments (Figure S10B and Fig. 4, respectively). Combinative EFTEM images of the ex situ heated sample up to $850^{\circ} \mathrm{C}$ highlighted an even distribution of $\mathrm{Ni}$ and $\mathrm{Fe}$ throughout the material. This was 

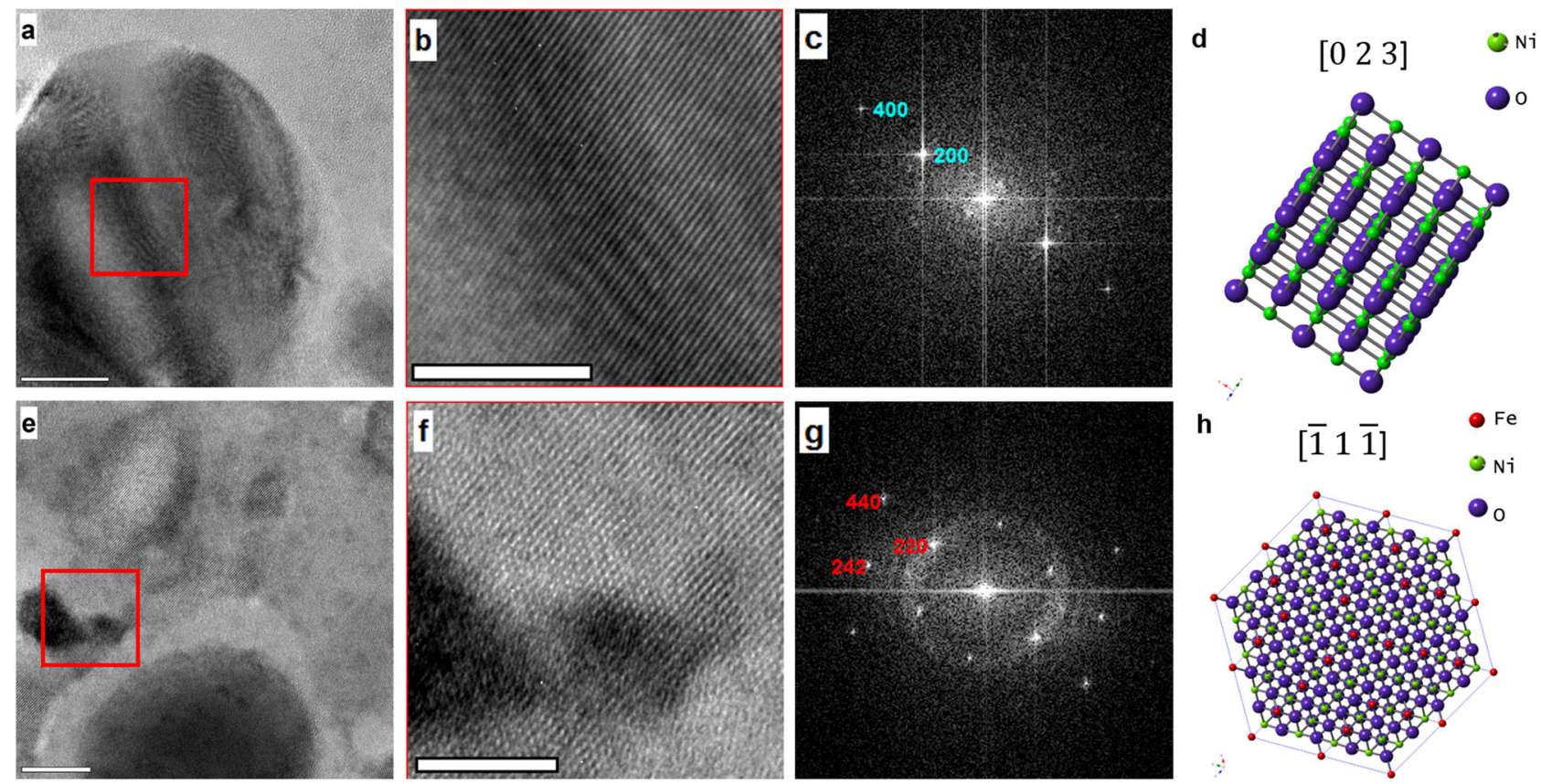

Fig. 4 a, e HRTEM images of various regions after in situ TEM heating experiments. $\mathbf{b}$, $\mathbf{f}$ Zoomed in images of square regions as indicated in a and e. $\mathbf{c}, \mathbf{g}$ Calculated FFT from square red region as indicated on the TEM micrograph in a and e. Blue and red annotations (c and $\mathbf{g}$ ) represent labelled $\mathrm{NiO}$ and trevorite crystallographic planes, respectively. $\mathbf{d}, \mathbf{h}$ Schematic representations of the respective crystalline structures, viewed along the [023] (d) and [111] (h) directions. Scale bars are $10 \mathrm{~nm}$ for micrographs a and e and $5 \mathrm{~nm}$ for $\mathbf{b}$ and $\mathbf{f}$. Structures $\mathbf{d}$ and $\mathbf{h}$ were visualised using CrystalMaker ${ }^{T M}$

evidenced by the ubiquitous EFTEM intensities of $\mathrm{Ni}$ (green) and Fe (red), respectively (Figure S9A). The non-specific location of either $\mathrm{Ni}$ or Fe contrasts the EFTEM findings from in situ experiments (Fig. 3a). This is acknowledged to be the effect of rehydration of the heated $\mathrm{LDH}$ material due to its exposure to moist air conditions as a consequence of sample preparation, where the $\mathrm{Ni}$ and Fe sites could revert back to an LDH structure. A potential reconstruction to an LDH structure was also verified by STEM-EDX, demonstrating a greater Ni content in the analysed region (areas 3 and 4 in Figure S8B and Table 2 in Supporting Information).

Despite maintaining the focus of interest on the nanoscale features responsible for the thermal evolution of these LDH samples, it is acknowledged that the above findings may not have reflected their bulk material counterparts. Hence, we compared these behaviours via the application of in situ and ex situ XRD analysis (Figure S11A and B, respectively). Powder samples were measured at intermediate temperature stages of aforementioned heating protocols (Figure S2). In particular, XRD was strategically conducted firstly at room temperature and then samples were heated to $250^{\circ} \mathrm{C}$ for $2 \mathrm{~h}$ followed by $450^{\circ} \mathrm{C}$ for $2 \mathrm{~h}$ and finally to $850^{\circ} \mathrm{C}$ for $2 \mathrm{~h}$ and post-analysed at each stage (Figure S11A(a)-(d) and Figure $\mathrm{S} 11 \mathrm{~B}(\mathrm{a})-(\mathrm{d})$, respectively). At room temperature, the in situ XRD analysis revealed typical LDH (003), (006), (101), (015) and (110) crystallographic planes at $2 \theta$ angles $16^{\circ}, 26.6^{\circ}, 39.4^{\circ}$, $45.7^{\circ}$ and $70.9^{\circ}$, respectively. ${ }^{48}$ Upon thermal treatment to $250^{\circ} \mathrm{C}$, a lateral shift of the (003) and loss of (006) basal planes suggests an expansion of the unit cell and a reduction in basal plane periodicity. This is attributed to the dehydration of the associated $\mathrm{H}_{2} \mathrm{O}$ content from interlayer LDH galleries. Moreover, the retention of the LDH (110) plane is observed in both in situ TEM and in situ XRD approaches (Fig. $2 g$ and Figure S11A(b)), validating how single nanoparticle behaviours can affect bulk material characteristics. As the samples were subject to $450^{\circ} \mathrm{C}$ in situ, a phase transition took place. The loss of the LDH diffraction peaks and the appearance of the (111), (200) and (220) of $\mathrm{NiO}$ occurred at $43.4^{\circ}$, $50.6^{\circ}$ and $74.1^{\circ}$, respectively. ${ }^{49}$ This behaviour was also viewed via the in situ TEM experiments, where the birth of the spherical $\mathrm{NiO}$ particles was observed at this temperature (Fig. 2C). At $850^{\circ} \mathrm{C}$, the (111), (200) and (220) diffraction peaks of $\mathrm{NiO}$ became more pronounced. This is believed to be due to the growth and nucleation of further $\mathrm{NiO}$ particles, as observed in our in situ TEM experiments (Fig. 2d). It is also possible that these diffraction peaks could be attributed to (222), (400) and (440) of the $\mathrm{NiFe}_{2} \mathrm{O}_{4}$ phase, but this is difficult to distinguish due to their ambiguity with the $\mathrm{NiO}$ peaks. Parallel ex situ XRD experiments were also carried out on the $\mathrm{Ni}-\mathrm{Fe} \mathrm{LDH}$ samples. At room temperature, the XRD peaks at $2 \theta$ angles $5.3^{\circ}, 10.32^{\circ}, 15.7^{\circ}, 17.6^{\circ}$ and $20.7^{\circ}$ corresponded to the (003), (006), (101), (104) and (018) planes of the Ni-Fe LDH crystallographic planes (Figure $\mathrm{S} 11 \mathrm{~B}(\mathrm{a})) .^{50}$ Similar traits relative to in situ XRD approaches were evidenced at $250^{\circ} \mathrm{C}$. The loss of the (006), (101), (104) and (018) peaks indicate a breakdown in the $\mathrm{Ni}$ Fe LDH crystal structure. At this temperature, the XRD patterns also showed a decreasing and broadening of the (003) peaks indicating an alteration in basal spacings (Figure S11B (b)). The evolution of the $\mathrm{NiO}(111),(200)$ and (220) planes and the $\mathrm{NiFe}_{2} \mathrm{O}_{4}$ (220), (311), (222), (400), (511), (440), (622) and (444) planes were evidenced at $450^{\circ} \mathrm{C}$ and $850^{\circ} \mathrm{C}$, respectively (Figure S11B(c) and (d) respectively). The evolution of these $\mathrm{NiO}$ phases is also mirrored via our in situ XRD approach. Moreover, the NiO $\{200\}$ and $\mathrm{NiFe}_{2} \mathrm{O}_{4}\{220\}$ planar families were observed both in the SAED patterns recorded during in situ TEM experiments (Figs. $2 \mathrm{~h}$ and $4 \mathrm{f}$, respectively). However, ambiguities were also seen in selected ex situ XRD peaks of the calcined samples as they could correspond to either oxide or trevorite structures. The XRD peaks at $2 \theta$ angles $17.0^{\circ}, 19.7^{\circ}$ and $27.9^{\circ}$ could be hypothesised to derive from the respective $\mathrm{NiO}$ (111), (200) and (220) or $\mathrm{NiFe}_{2} \mathrm{O}_{4}(222)$, (400) and (440) family of planes. This has also been observed by previous work. ${ }^{22}$ Furthermore, this indistinctness in the possible source of XRD peaks validates our motivation of applying the 

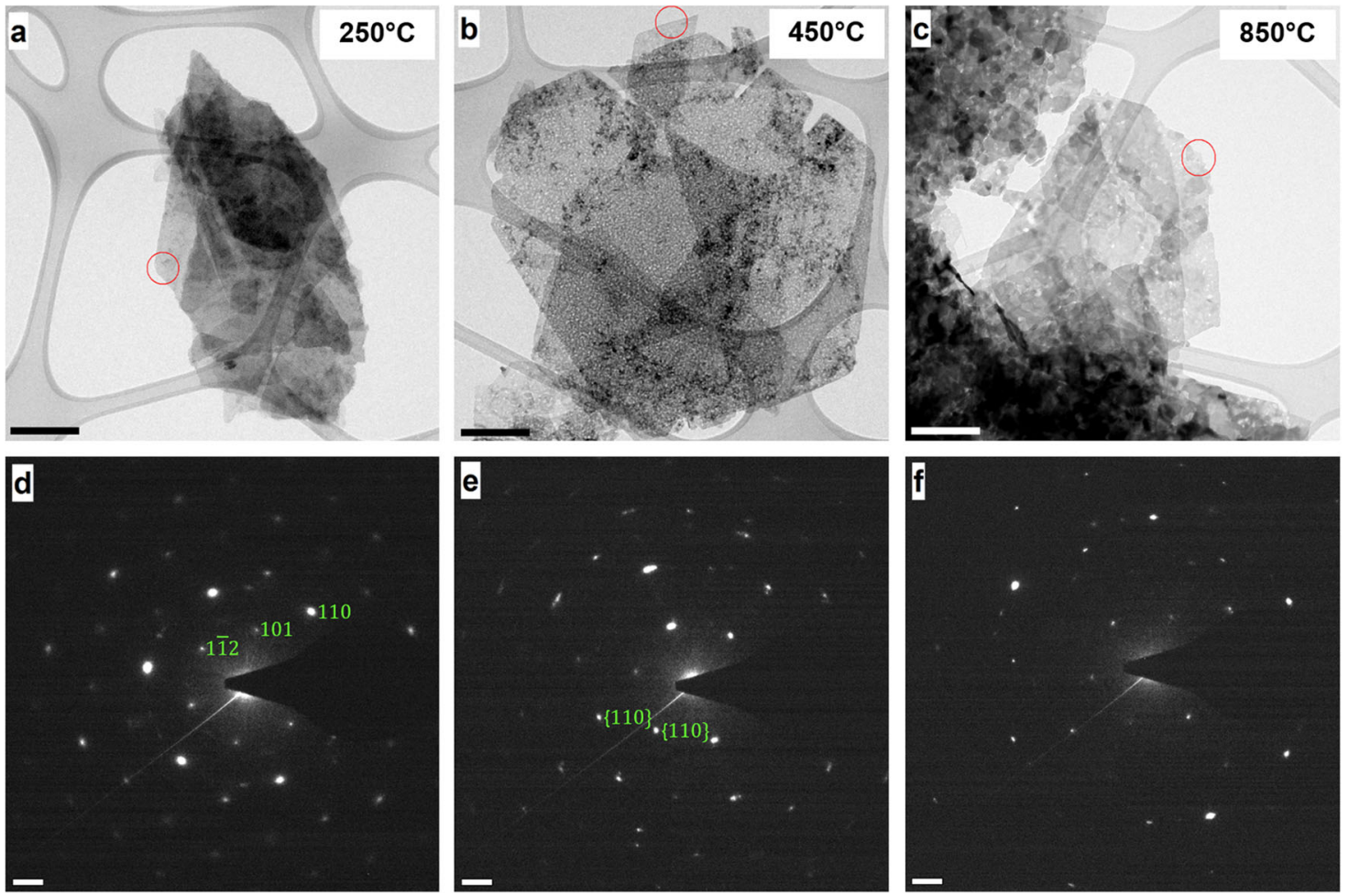

Fig. 5 BFTEM and associated SAED patterns of ex situ heating experiments of the Ni-Fe LDH. a-c BFTEM and d-f SAED patterns corresponding to the end of the $250^{\circ} \mathrm{C}$ step, end of the $450^{\circ} \mathrm{C}$ step and end of the $850^{\circ} \mathrm{C}$ step of the applied heating ramp, respectively. Green annotations (d and e) represent labelled LDH crystallographic planes. Scale bars for TEM micrographs a-c and SAED patterns d-f are $200 \mathrm{~nm}$ and $2 \mathrm{~nm}{ }^{-1}$, respectively

techniques of SAED and HRTEM. The observed correlations between XRD macroscopic techniques and EM analysis indicates that the bulk features derived from the LDH nanoscale transformations. Hence, our findings highlight the importance of understanding the nanoscale thermal degradation properties for the design and fabrication of LDH-based devices.

In light of such findings related to the $\mathrm{Ni}-\mathrm{Fe} \mathrm{LDH}$ material, we applied an identical experimental approach and analysis to a similar Mg-Al LDH structure. This yielded a comparison of both size and composition of various LDH structures with regards to their associated thermal degradations. Figure 6 displays the TEM findings from in situ heating of an Mg-AI LDH material, where the aforementioned heating ramp was imposed (Figure S2). Similar to the $\mathrm{Ni}-\mathrm{Fe} \mathrm{LDH}$, the material appeared to have a well-defind platelet structures; however, they portrayed a width of approximately $350 \mathrm{~nm}$ and rounded morphology, as shown by BFTEM (Fig. 6a). Moreover, the crystallographic resemblances of these hydrotalcite platelets to the $\mathrm{Ni}$-Fe compositions were seen via the existence of well-defined $\{100\},\{110\},\{120\}$ and $\{330\}$ planar families of the LDH structure (Fig. 6e)..$^{51}$

An overall examination of the LDH platelets as they are subject to the in situ heating ramp revealed an inhomogeneous contrast and a porous structure develops within the platelets (Fig. $6 \mathrm{~d}$ and Movie V2 Supplementary Information). These porous structures evolved in an irregular, somewhat random, fashion on the hydrotalcite materials. The borders of the platelets were not observed to have the same behaviours, possibly due to their different crystallographic environments when compared the central domains of the materials. In contrast, they developed a denser frame on the outer regions of the heated material. Indeed, it is indicative that the composition and dimension of LDH materials has an influential effect on its thermal degradative properties. Similar features of the Mg-Al LDH degradation, in particular the evolution of a porous matrix, are also evidenced in the recent independent studies of Warringham et al. ${ }^{52}$ In contrast to the Mg-Al LDH thermal degradation, the Ni-Fe LDH does not develop a morphological variation along the borders of the platelets (Figs. $6 \mathrm{~d}$ and $2 \mathrm{~d}$, respectively). Analogously, the development of a porous structure of the Ni-Fe LDH is not as pronounced as the Mg-Al counterpart. The Mg-Al LDH morphology and crystalline structures are maintained as they are subject to in situ heating up to $250^{\circ} \mathrm{C}$, as demonstrated by the TEM micrographs and electron diffraction patterns in Fig. 6b, f. An intensity attenuation in the $\{100\}$ and $\{110\}$ diffraction spots occurs (Fig. 6f). The loss in periodicity of these planes suggests a crystallographic rearrangement of the $\mathrm{LDH}$ structures and is accredited to the loss of $\mathrm{H}_{2} \mathrm{O}$ from the interstitial layers and a dehydroxlisation of the octahedral layers. ${ }^{23,53}$ Interestingly, this transformation was also paralleled in the Ni-Fe LDH structures at this temperature (Fig. 2f).

As the continuation of the in situ heating ramp regime reached $450^{\circ} \mathrm{C}, \mathrm{Mg}$-Al platelets demonstrated increased porosity with an evident rearrangement taking place, as shown in BFTEM (Fig. 6C). The overall morphology of the $\mathrm{LDH}$ remained intact but an amorphization occurred. This was evidenced by in situ SAED patterns via the loss of intensity and broadening of the $\{100\},\{110\}$ and $\{120\}$ LDH diffraction spots (Fig. 6f, g). Moreover, there are parallels in this Mg-Al LDH crystallographic transition, however, as amorphization was found to occur at approximately $450^{\circ} \mathrm{C}$ when compared to the $\mathrm{Ni}-\mathrm{Fe}$ degradations, which displayed an amorphization tendencies at $250^{\circ} \mathrm{C}$ (Fig. 2f). Hence, it is believed that the Mg-Al LDH structural transformations are less susceptible to degradations at higher temperatures, suggesting that LDH dehydration procedures exhibit a compositional dependence. This 

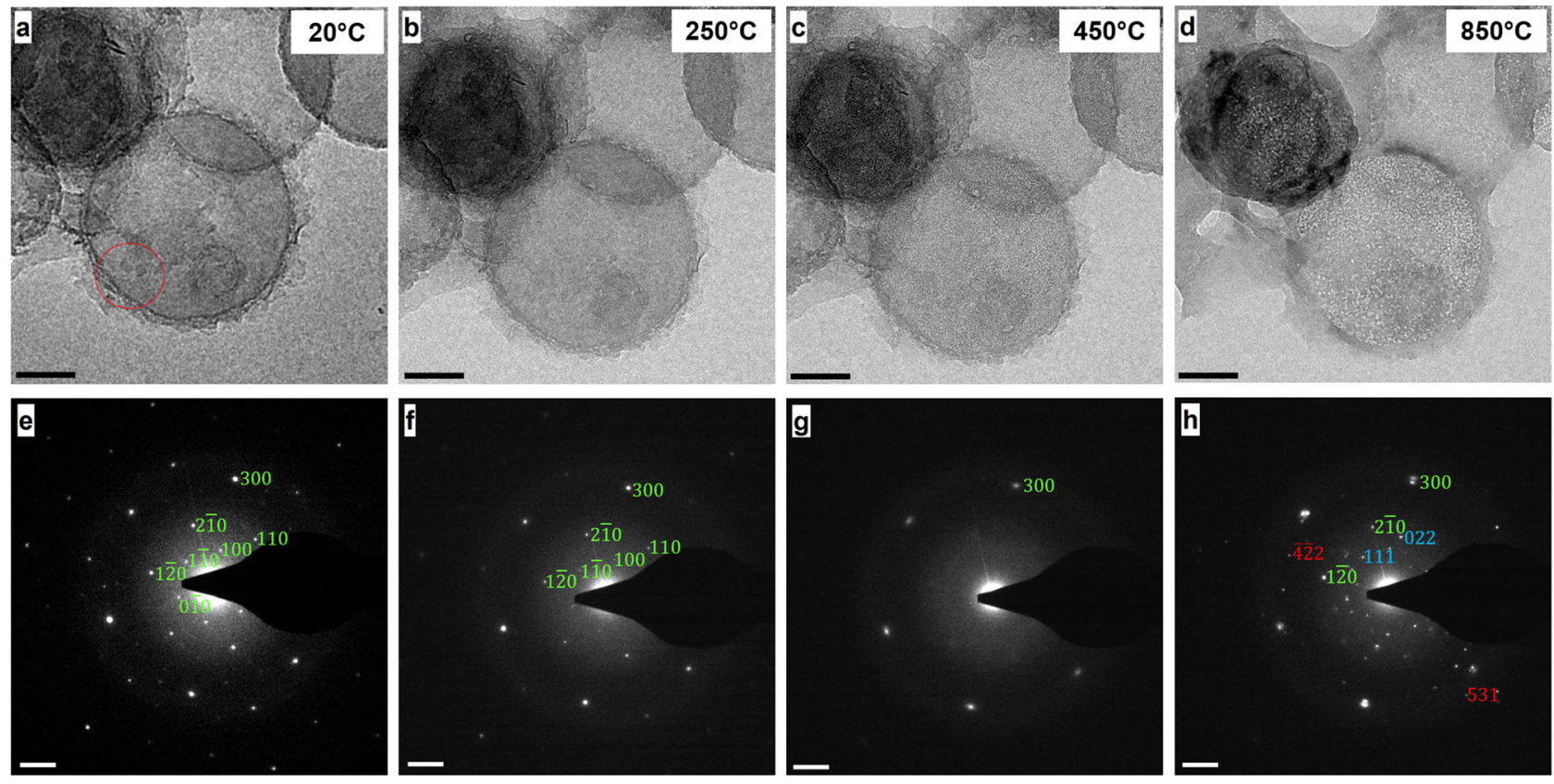

Fig. 6 BFTEM and associated SAED patterns from various stages of the in situ heating experiments of the Mg-Al LDH. a-d BFTEM and e-h SAED patterns corresponding to column temperature $20^{\circ} \mathrm{C}$, end of $250^{\circ} \mathrm{C}$ step, end of $450^{\circ} \mathrm{C}$ step and end of $850{ }^{\circ} \mathrm{C}$ step, respectively. Scale bars are $100 \mathrm{~nm}$ for images $\mathbf{a}-\mathbf{d}$ and are $2 \mathrm{~nm}^{-1}$ for $\mathbf{e}-\mathbf{h}$

feature was also corroborated in our TGA, where greater mass losses were observed in the $\mathrm{Ni}$-Fe case (Figure $\mathrm{S} 12$ ).

At the $850^{\circ} \mathrm{C}$ step, the emergence of new diffraction spots in the associated SAED patterns provided direct experimental confirmation of a crystallographic transformation from an LDH material to a combination of $\mathrm{a}^{-} \mathrm{Al}_{2} \mathrm{O}_{3}$ and $\mathrm{MgAl}_{2} \mathrm{O}_{4}$ spinel structures (Fig. 6h). This was evidenced by the existence of the associated $\{111\}$ and $\{022\}$ reflections of the $\mathrm{a}-\mathrm{Al}_{2} \mathrm{O}_{3}$ and $\{422\}$, $\{531\}$ and $\{444\}$ of $\mathrm{MgAl}_{2} \mathrm{O}_{4}$ spinel. ${ }^{54}$ This evolution to mixed metal oxides was paralleled in the $\mathrm{Ni}$-Fe degradations at this temperature (Fig. 2h) and was also similar to previous work. ${ }^{24,55}$ These in situ methods showed that a complete decomposition did not occur via the existence of the $\{110\},\{120\}$ and $\{300\}$ LDH planes (Fig. 6h).

The size dependence of the LDH platelets to their calcination behaviours was also investigated (Figure S14). We applied our in situ heating experiment conditions to Mg-Al LDH platelets of relatively larger size (approximately $3 \mu \mathrm{m}$ in lateral dimension). In comparison to the smaller Mg-Al LDH material (Fig. 6), the larger platelets were seen to have very similar degradation procedures. The reduction in crystallinity up to $450^{\circ} \mathrm{C}$ (Figure S14E-G) and evolution of pores across the material at $850^{\circ} \mathrm{C}$ (Figure S14D) compares to that of their smaller counterparts (Fig. 6). Overall, it was seen that the degradations were not platelet size dependent, rather the materials composition having a more profound effect on such mechanistic behaviours.

Our results yield important impacts in relation to the employment of LDHs as dye adsorbents, flame retardants and catalysts. For example, our findings potentially impart further information into the role of LDH platelets and their calcined derivatives have on the thermal stability, toxicity and structural morphology of when incorporated into polymers or resins. ${ }^{11}$ Moreover, the distribution of calcined products could be of interest for the design and testing of future nanocatalysts in relation to the dispersion and confinement of catalytic sites with enhanced catalytic activity or selectivity. The impacts of our analysis may further be extended to dye sorbents, possibly revealing insights as to how sorption capacities are affected by the extent of LDH calcinations. More generally, the application of in situ techniques elucidated the progressions of the structural alterations of the nanoplatelets. We have revealed a direct observation of the nanoscale nucleation behaviours and mechanisms involved in $\mathrm{Ni}$ Fe LDH and Mg-Al LDH thermal degradation.

This study reports the application of in situ TEM to investigate and compare the thermal degradation behaviours of LDH materials. The application of in situ heating techniques in the TEM revealed the morphological and crystallographic integrity of individual LDH platelets as well as the nanoscale behaviours and progression mechanisms involved in Mg-AI LDH thermal degradation. Ni-Fe and Mg-Al LDH compounds exhibited similar behaviours but also had unique signatures in their own right. The in situ heating of $\mathrm{Ni}-\mathrm{Fe} \mathrm{LDH}$ material resulted in the transformation into a coexistence of $\mathrm{Ni}$ oxide-type particles arranged throughout a $\mathrm{NiFe}_{2} \mathrm{O}_{4}$ trevorite matrix, confirmed by TEM, HRTEM and STEM-EDX. In particular, the nucleation processes of $\mathrm{NiO}$ particles was directly visualised by EFTEM methods. The calcinations were also paralleled via ex situ and XRD methods, exhibiting identical phase transformations, verifying the observed behaviours by TEM are due to the inherent properties of the LDH materials. It was found that the calcined Mg-Al LDH evolved into $\mathrm{Al}_{2} \mathrm{O}_{3}$ and $\mathrm{MgAl}_{2} \mathrm{O}_{4}$ structures, effectively characterised by HRTEM and SAED. However, the transition of the Mg-Al material occurred via a different mechanism, with a development of a porous matrix as opposed to the generation of spherical particles. The application of SAED in situ effectively characterised the crystallographic transitions involved in the degradations of both $\mathrm{Ni}-\mathrm{Fe}$ and $\mathrm{Mg}-\mathrm{Al}$ of $\mathrm{LDH}$ materials.

The applicability of our experimental methodologies was confirmed by our analysis of LDH materials of a different compositions, establishing a suitable in situ approach to characterise the LDH thermal transformation behaviours. Ultimately, our successful nanoscale assessment of such mechanisms has an important bearing on their further development and strategic designs of catalysts and flame retardants as well as many additional current areas of nanotechnology. 


\section{METHODS}

$\mathrm{Ni}$-Fe LDH synthesis

$\mathrm{Ni}\left(\mathrm{NO}_{3}\right)_{2} \cdot 6 \mathrm{H}_{2} \mathrm{O}, \mathrm{Fe}\left(\mathrm{NO}_{3}\right)_{3} \cdot 9 \mathrm{H}_{2} \mathrm{O}$, TEA and urea were dissolved in $80 \mathrm{ml}$ of deionized water giving a final concentration of $7.5,2.5,10$ and $17.5 \mathrm{mM}$, respectively, and stirred at room temperature for $24 \mathrm{~h}$. Then, the reaction mixture was heated to $100^{\circ} \mathrm{C}$ in an oil bath for $48 \mathrm{~h}$ and the obtained yellow precipitate was washed with deionized water several times.

\section{Mg-Al LDH synthesis}

To prepare a 3:1 Mg-Al LDH with intercalated carbonate, $343 \mathrm{~g}(1.68 \mathrm{~mol})$ $\mathrm{MgCl}_{2} \cdot 6 \mathrm{H}_{2} \mathrm{O}$ and $136 \mathrm{~g}(0.56 \mathrm{~mol}) \mathrm{AlCl}_{3} \cdot 6 \mathrm{H}_{2} \mathrm{O}$ were dissolved in $100 \mathrm{ml}$ deionized water as reactant mixture $\mathrm{A}$. $\mathrm{A}$ second reactant mixture $\mathrm{B}$ was prepared by dissolving $180 \mathrm{~g}(4.5 \mathrm{~mol}) \mathrm{NaOH}$ pellets and $30 \mathrm{~g}(0.28 \mathrm{~mol})$ $\mathrm{Na}_{2} \mathrm{CO}_{3}$ in $100 \mathrm{ml}$ deionized water. Subsequently, the reactant mixtures $\mathrm{A}$ and $B$ were pumped together within 30 min with a syringe pump into a beaker, containing another $150 \mathrm{ml}$ deionized water, under heavy shearmixing using an ultraturrax rotor-stator-mixer. The reaction product was subsequently washed five times with deionized water via dispersing the particles in fresh deionized water and solid-liquid separating the particles via gravitational sedimentation (centrifugation). Eventually, the whole product of the washed LDH particles was redispersed in $1,500 \mathrm{ml}$ of deionized water and heated at $80^{\circ} \mathrm{C}$ for 4 days to yield well-ordered crystallites.

To synthesise large platelets of carbonate containing Mg-Al LDH with Mg:Al ratio of 3, $\mathrm{Mg}\left(\mathrm{NO}_{3}\right)_{2} \cdot 6 \mathrm{H}_{2} \mathrm{O}, \mathrm{Al}\left(\mathrm{NO}_{3}\right)_{3} \cdot 9 \mathrm{H}_{2} \mathrm{O}$ and urea were dissolved in $60 \mathrm{ml}$ of deionised water giving a final concentration of $0.125,0.042$ and $1.67 \mathrm{M}$, respectively. Then, the solution was transferred to $100 \mathrm{ml}$ round bottom flask and immersed in an oil bath previously heated to $90^{\circ} \mathrm{C}$ and continuously stirred for $48 \mathrm{~h}$ under reflux. After this time, the flask was cooled in a water bath for $30 \mathrm{~min}$, the precipitate was separated by centrifugation $(3,000 \mathrm{rpm} / 10 \mathrm{~min})$ and washed three times with water.

\section{Experimental methods}

Heating conditions of $L D H$ materials for in situ and ex situ experiments. The $\mathrm{LDH}$ samples were initially exposed to a temperature of $30^{\circ} \mathrm{C}$ for $10 \mathrm{~min}$. This was followed by a ramp to $250^{\circ} \mathrm{C}$ at $10^{\circ} \mathrm{C} / \mathrm{min}$ and kept at this temperature for $120 \mathrm{~min}$. Subsequently, the same sample was heated to $450^{\circ} \mathrm{C}$ at $10^{\circ} \mathrm{C} / \mathrm{min}$ and kept at $450^{\circ} \mathrm{C}$ for $120 \mathrm{~min}$. Finally, the sample was elevated to $850^{\circ} \mathrm{C}$ at $10^{\circ} \mathrm{C} / \mathrm{min}$ and maintained at $850^{\circ} \mathrm{C}$ for $2 \mathrm{~h}$.

\section{TEM sample preparation}

Samples were prepared for in situ TEM experiments by suspending the synthesised LDH materials in deionized water and sonicating for $30 \mathrm{~min}$. Subsequently, $5 \mu \mathrm{l}$ of the associated samples were subsequently placed DENS Solutions Nano-chips ${ }^{\mathrm{TM}}$ for in situ experiments, respectively. Excess sample was wicked using filter paper and then air dried for approximately 30 min each before TEM analysis. Ex situ heated Ni-Fe samples were prepared for TEM analysis by directly depositing the powder forms of the materials onto lacey Carbon TEM Cu grids (TED Pella Inc., USA).

\section{Characterisation methods}

Powder XRD was used to characterise the precursor LDH bulk sample as well as LDH samples subject to various thermal conditions. A Bruker Advance Powder X-ray diffractometer (Bruker, MA, USA) operating with a molybdenum K-a emission source $\left(\lambda=0.7107 \AA^{-1}\right)$ was used to record the associated diffractograms. In situ XRD experiments were conducted using a PANalytical EMRYREAN XRD with $\theta-\theta$ Bragg-Brentano geometry. A cobalt K- $a_{1,2}$ emission source $\left(\lambda=1.789 \AA^{-1}\right)$. To exclude any possibility of reconstruction to an LDH structure due to water exposure, we performed XRD using samples in powder form.

TGA was also conducted as a measure of the mass losses when the LDH was subject to thermal environments. TGA studies were performed using a PerkinElmer Pyris 1 TGA (PerkinElmer, MA, USA).

TEM was used to characterise the overall morphology and crystallographic structure of the associated LDH samples. TEM and SAED were conducted on a FEl Titan300 (FEl, Oregon, USA), operated at $300 \mathrm{kV}$. For comparative purposes of the in situ heating experiments, TEM magnifications and electron diffraction camera lengths were kept constant. In situ heating TEM experiments were conducted using a DENS Solutions Wildfire $^{\mathrm{TM}}$ TEM holder (DENS solutions, Delft, The Netherlands). This experimental in situ TEM sample holder set-up consists of a microelectromechanical systems chip design with electron transparent silicon nitride windows onto which the sample is deposited. The heating of the chip is conducted via a metallic spiralling system with suitable contacts and interpolated with an electron transparent SiN window support film, onto which the sample is directly deposited. Hence this set-up exposes the sample to thermal environments, and as such, the nanoscale properties and features of the LDH materials can be characterised as they are subject to specific elevated temperatures. Furthermore, the TEM set-up was carefully monitored to reduce the beam effects discussed above. As the sample was subject to heating, the electron beam was blanked to avoid unwarranted interactions. By doing this, we can be reassured that the discussed findings are indeed effect of the applied heating treatment rather than undesired beam-induced effects. EDX and EFTEM studies were carried out using a EDAX single SiLi EDX detector and GIF Tridiem energy filter (Gatan, USA) with a spectrometer, respectively. Our SAED experimental set-up utilised a selected area aperture to assist in measuring the relative diffraction patterns, allowing us to analyse crystallographic properties from regions much smaller than the individual hydrotalcites themselves. An analysis of such materials exposed to in situ heating environments yielded a direct visualisation of how these elevated temperatures affect the properties of LDH materials. In addition, the accompanying SAED methods permitted an investigation into the crystallographic features that occur at the various thermal stages in real time. This approach was used to directly observe the thermal degradations of hydrotalcites and assess the nanoscale features that play a significant role in these processes such as size, morphology and localised composition.

TEM images and SAED patterns were analysed using Digital Micrograph (Gatan Inc., California, USA). The EFTEM experimental technique extends from EELS in the electron microscope. Through collection and analysis of the inelastically scattered electrons transmitted through the sample, an EEL spectrum can be acquired. At higher energies of these spectra, coreloss signals in EELS originate from the ionisation of inner shells (e.g. K, L or M) due to energy transfer from the incident electron beam, and are characteristic of which element they come from. By selecting certain energies using an energy slit width to contain such ionisation edge signals, the unwanted electrons are filtered out, resulting in images containing vital elemental information. ${ }^{56}$

Data availability

The data related to the findings of this work are available from the corresponding author subject to reasonable request.

\section{ACKNOWLEDGEMENTS}

We would like to acknowledge the following funding supports: SFI AMBER, SFI PIYRA, ERC StG 2D NanoCaps, ERC CoG 3D2DPrint and Horizon2020 NMP Co-Pilot. We would like to thank the Advanced Microscopy Laboratory at CRANN, Trinity College Dublin. The Microanalytical Laboratory, School of Chemistry, University College Dublin is acknowledged for bulk ICP-AAS analysis.

\section{AUTHOR CONTRIBUTIONS}

C.H., K.M., M.C.D.M. and V.N. discussed the proposed plans of this work. S.J. and M.C.D. $M$ synthesised the associated Ni-Fe LDH and Mg-Al LDH samples, respectively. C.H. and E.K.MCC. performed in situ TEM characterisation experiments. C.H. and C.D. performed post in situ analysis via (S)TEM methods. C.H. performed ex situ analysis using TEM methods. S.J. performed ex situ heating experiments along with XRD and TGA characterisations. K.O. and K.G. performed in situ XRD experiments C.H., M.C.D.M. and A.S. interpreted electron diffraction results. C.H., K.M. and V.N. discussed and structured the paper content and results. C.H. wrote the paper with all authors contributing to preparation of the manuscript.

\section{ADDITIONAL INFORMATION}

Supplementary information accompanies the paper on the npj 2D Materials and Applications website (https://doi.org/10.1038/s41699-018-0048-4).

Competing interests: The authors declare no competing financial interests.

Publisher's note: Springer Nature remains neutral with regard to jurisdictional claims in published maps and institutional affiliations. 


\section{REFERENCES}

1. Coleman, J. N. et al. Two-dimensional nanosheets produced by liquid exfoliation of layered. Mater. Sci. (80-.). 331, 568-571 (2011).

2. Cavani, F., Trifiro, F. \& Vaccari, A. Hydrotalcite-type anionic clays: preparation, properties and applications. Catal. Today 11, 173-301 (1991).

3. Xu, Z. P. et al. Subcellular compartment targeting of layered double hydroxide nanoparticles. J. Control Rel. 130, 86-94 (2008).

4. Han, N., Zhao, F. \& Li, Y. Ultrathin nickel-iron layered double hydroxide nanosheets intercalated with molybdate anions for electrocatalytic water oxidation †. J. Mater. Chem. A 3, 16348-16353 (2015).

5. Fan, G., Li, F., Evans, D. G. \& Duan, X. Catalytic applications of layered double hydroxides: recent advances and perspectives. Chem. Soc. Rev. 43, 7040-7066 (2014).

6. Chen, H., Hu, L., Chen, M., Yan, Y. \& Wu, L. Nickel-cobalt layered double hydroxide nanosheets for high-performance supercapacitor electrode materials. Adv. Funct. Mater. 24, 934-942 (2014).

7. Ulibarri, M. A., Pavlovic, I., Barriga, C. \& Hermosın, M. C. Adsorption of anionic species on hydrotalcite-like compounds: effect of interlayer anion and crystallinity. Appl. Clay Sci. 18, 17-27 (2001).

8. Yan, D. et al. In situ polymerization of the 4-vinylbenzenesulfonic anion in Ni-Allayered double hydroxide and its molecular dynamic simulation. J. Phys. Chem. A 112, 7671-7681 (2008).

9. Zhao, Y., Hu, H., Yang, X., Yan, D. \& Dai, Q. Tunable electronic transport properties of $2 \mathrm{D}$ layered double hydroxide crystalline microsheets with varied chemical compositions. Small 12, 4471-4476 (2016).

10. Matusinovic, Z. \& Wilkie, C. A. Fire retardancy and morphology of layered double hydroxide nanocomposites: a review. J. Mater. Chem. 22, 18701-18704 (2012)

11. Gao, Y., Wu, J., Wang, Q., Wilkie, A. \& Hare, D. O. Flame retardant polymer/layered double hydroxide nanocomposites. J. Mater. Chem. A 2, 10996-11016 (2014).

12. Yan, D. et al. A combined study based on experiment and molecular dynamics: perylene tetracarboxylate intercalated in a layered double hydroxide matrix. Phys. Chem. Chem. Phys. 11, 9200-9209 (2009).

13. Li, C., Wei, M., Evans, D. G. \& Duan, X. Recent advances for layered double hydroxides (LDHs) materials as catalysts applied in green aqueous media. Catal. Today 247, 163-169 (2015).

14. Shan, X., Song, L., Xing, W., Hu, Y. \& Lo, S. Effect of nickel-containing layered double hydroxides and cyclophosphazene compound on the thermal stability and flame retardancy of poly(lactic acid). Ind. Eng. Chem. Res. 51, 13037-13045 (2012).

15. Lu, Y. et al. Highly sensitive nonenzymatic glucose sensor based on 3D ultrathin NiFe layered double hydroxide nanosheets. Electroanalysis 29, 1755-1761 (2017).

16. Gong, M. \& Dai, H. A mini review of NiFe-based materials as highly active oxygen evolution reaction electrocatalysts. Nano Res. 8, 23-39 (2015).

17. Gong, M. et al. An Advanced Ni-Fe layered double hydroxide electrocatalyst for water oxidation. J. Am. Chem. Soc. 135, 8452-8455 (2013).

18. Dionigi, F., Reier, T., Pawolek, Z., Gliech, M. \& Strasser, P. Design criteria, operating conditions, and nickel-iron hydroxide catalyst materials for selective seawater electrolysis. ChemSusChem 9, 962-972 (2016).

19. Flegler, A., Müssig, S., Prieschl, J., Mandel, K. \& Sextl, G. Towards core-shell bifunctional catalyst particles for aqueous metal-air batteries: NiFe-layered double hydroxide nanoparticle coatings on g-MnO2 microparticles. Electrochim. Acta 231, 216-222 (2017).

20. Sjåstad, A. O., Andersen, H., Vajeeston, P., Karthikeyan, J. \& Arstad, B. On the thermal stability and structures of layered double hydroxides $\mathrm{Mg} 1-x$ Alx $(\mathrm{OH}) 2$ (NO3) $x \cdot \mathrm{mH} 2 \mathrm{O}(0.18<x<0$.38). Eur. J. Inorg. Chem. 2, 1775-1788 (2015).

21. Kobayashi, Y., Ke, X., Hata, H., Schiffer, P. \& Mallouk, T. E. Soft chemical conversion of layered double hydroxides to superparamagnetic spinel platelets. Chem. Mater. 20, 2374-2381 (2008).

22. Xiao, T., Tang, Y., Jia, Z., Li, D. \& Hu, X. Self-assembled 3D flower-like $\mathrm{Ni}^{2+}-\mathrm{Fe}^{3+}$ layered double hydroxides and their calcined products. IOP Nanotechnol. 20, 475603 (2009).

23. Mokhtar, M., Inayat, A., Ofili, J. \& Schwieger, W. Thermal decomposition, gas phase hydration and liquid phase reconstruction in the system $\mathrm{Mg} / \mathrm{Al}$ hydrotalcite/ mixed oxide: a comparative study. Appl. Clay Sci. 50, 176-181 (2017).

24. Costa, D. G., Rocha, A. B., Souza, W. F., Chiaro, S. S. X. \& Leita, A. A. Ab initio study of reaction pathways related to initial steps of thermal decomposition of the layered double hydroxide compounds. J. Phys. Chem. C 116, 13679-13687 (2012).

25. Valente, J. S. et al. Basic properties of the mixed oxides obtained by thermal decomposition of hydrotalcites containing different metallic compositions. J. Catal. 381, 370-381 (2000).

26. Lei, X. et al. Activated MgAl-layered double hydroxide as solid base catalysts for the conversion of fatty acid methyl esters to monoethanolamides. Appl. Catal. A 399, 87-92 (2011).
27. Perez-Ramirez, J., Mul, G., Kapteijn, F. \& Moulijn, J. A. In situ investigation of the thermal decomposition of $\mathrm{Co} \pm \mathrm{Al}$ hydrotalcite in different atmospheres. J. Mater. Chem. 11, 821-830 (2001).

28. Fan, Q. et al. Double-confined nickel nanocatalyst derived from layered double hydroxide precursor: atomic scale insight into microstructure evolution. ACS Chem. Mater. 28, 6296-6304 (2016).

29. Liang, R., Tian, R., Liu, Z., Yan, D. \& Wei, M. Preparation of monodisperse ferrite nanocrystals with tunable morphology and magnetic properties. Chem. - Asian J. 9, 1161-1167 (2014).

30. Abelló, S., Verboekend, D., Bridier, B. \& Pérez-ramírez, J. Activated takovite catalysts for partial hydrogenation of ethyne, propyne, and propadiene. J. Catal. 259 85-95 (2008).

31. Zhao, Y. et al. Oxide-modified nickel photocatalysts for the production of hydrocarbons in visible light. Angew. Chem. Int. Ed. 55, 4215-4219 (2016).

32. Manzi-nshuti, C., Wang, D., Hossenlopp, M. \& Wilkie, C. A. Aluminum-containing layered double hydroxides: the thermal, mechanical, and fire properties of (nano) composites of poly (methyl methacrylate) t. J. Mater. Chem. 18, 3091-3102 (2008).

33. Puttaswamy, N. S. \& Kamath, P. V. Reversible thermal behaviour of layered double hydroxides: a thermogravimetric study. J. Mater. Chem. 7, 1941-1945 (1997).

34. Tsyganok, A. \& Ã, A. S. Incorporation of transition metals into Mg-Al layered double hydroxides: Coprecipitation of cations vs. their pre-complexation with an anionic chelator. J. Solid State Chem. 179, 1830-1841 (2006).

35. Vaysse, C., Guerlou-Demourgues, L. \& Delmas, C. Thermal evolution of carbonate pillared layered hydroxides with $(\mathrm{Ni}, \mathrm{L})(=\mathrm{Fe}, \mathrm{Co})$ based slabs: grafting or nongrafting of carbonate anions? Inorg. Chem. 41, 3831-3839 (2002).

36. Vijaikumar, S., Dhakshinamoorthy, A. \& Pitchumani, K. L-Proline anchored hydrotalcite clays: an efficient catalyst for asymmetric Michael addition. Appl. Catal. A Gen. 340, 25-32 (2008).

37. Dumbre, D. K. et al. Thermally decomposed mesoporous nickel iron hydrotalcite: an active solid-base catalyst for solvent-free Knoevenagel condensation. J. Colloid Interface Sci. 441, 52-58 (2015).

38. Zhou, T. et al. Preparation and characterization of layered precursor nickel-iron hydrotalcites and magnetic materials. Acta Chim. Sin. 60, 1078-1083 (2002).

39. Boukraa, F. B. D. S., Su, B. \& Bettahar, N. Nickel-iron layered double hydroxide (LDH): textural properties upon hydrothermal treatments and application on dye sorption. J. Hazard. Mater. 165, 206-217 (2009).

40. Mourad, M. C. D. et al. Activation and local structural stability during the thermal decomposition of Mg/Al-hydrotalcite by total neutron scattering. J. Mater. Chem. 21, 15479-15485 (2011).

41. Abell, G., Coronado, E., Marti-Gastaldo, C., Pinilla-Cienfuegos, E. \& Ribera, A. Hexagonal nanosheets from the exfoliation of $\mathrm{Ni}^{2+}-\mathrm{Fe}^{3+} \mathrm{LDHs}$ : a route towards layered multifunctional materials. J. Mater. Chem. 20, 7451-7455 (2010).

42. Egerton, R. F., Li, P. \& Malac, M. Radiation damage in the TEM and SEM. Micron 35, 399-409 (2004).

43. Forticaux, A., Dang, L., Liang, H. \& Jin, S. Controlled synthesis of layered double hydroxide nanoplates driven by screw dislocations. Nano Lett. 15, 3403-3409 (2015).

44. Hunter, B. M., Hieringer, W., Winkler, J. R., Gray, H. B. \& Mu, A. M. Effect of interlayer anions on [NiFe]-LDH nanosheet water oxidation activity. RSC Energy Environ. Sci. 23, 1734-1743 (2016).

45. Ahn, C. C. \& Krivanek, O. EELS Atlas: A Reference Collection of Electron Energy Loss Spectra Covering All Stable Elements (Warrendale, Gatan, 1983).

46. Rougier, A. \& Da Rocha, M. Electrochromism of non-stoichiometric NiO thin film: as single layer and in full device. Appl. Phys. A 122, 1-7 (2016).

47. Wong, F. \& Buchheit, R. G. Utilizing the structural memory effect of layered double hydroxides for sensing water uptake in organic coatings. Prog. Org. Coat. 51, 91-102 (2004).

48. Arco, M., Malet, P., Trujillano, R. \& Rives, V. Synthesis and characterization of hydrotalcites containing $\mathrm{Ni}$ (II) and Fe (III) and their calcination products. ACS Chem. Mater. 11, 624-633 (1999).

49. El-kemary, M., Nagy, N. \& El-Mehasseb, I. Nickel oxide nanoparticles: synthesis and spectral studies of interactions with glucose. Mater. Sci. Semicond. Process. 16, 1747-1752 (2013).

50. Abelló, S., Bolshak, E. \& Montané, D. Ni-Fe catalysts derived from hydrotalcite-like precursors for hydrogen production by ethanol steam reforming. Appl. Catal. A Gen. 450, 261-274 (2013).

51. Arakcheeva, A. V., Pushcharovskii, D. Y., Rastsvetaeva, R., Atencio, D. \& Lubman, G. $\mathrm{U}$. Crystal structure and comparative crystal chemistry of $\mathrm{Al}_{2} \mathrm{Mg}_{4}(\mathrm{OH}) 12$ (CO3) $3 \mathrm{H} 2 \mathrm{O}$, a new mineral from the hydrotalcite-manasseite group. Crystallogr. Rep. 41, 972-981 (1996).

52. Warringham, R. et al. Mapping the birth and evolution of pores upon thermal activation of layered hydroxides. ACS Chem. Mater. 29, 4052-4062 (2017). 
53. Constantino, V. R. L. \& Hnnavaia, T. J. Basic properties of $\mathrm{Mg} 2+1, \mathrm{Al} 3+$, layered double hydroxides intercalated by carbonate, hydroxide, chloride, and sulfate anions. Inorg. Chem. 34, 883-892 (1995).

54. Allmann, R. Magnesium aluminium carbonate hydroxide tetrahydrate: a discussion. Am. Mineral. 53, 1057 (1968).

55. Yang, W., Kim, Y., Liu, P. K. T., Sahimi, M. \& Tsotsis, T. T. A study by in situ techniques of the thermal evolution of the structure of a $\mathrm{Mg}-\mathrm{Al}^{-\mathrm{CO}_{3}}$ layered double hydroxide. Chem. Eng. Sci. 57, 2945-2953 (2002).

56. Willams, D. B. \& Carter, C. B. Transmission Electron Microscopy: A Textbook for Materials Science. Springer, Boston, MA, USA (2009).

Open Access This article is licensed under a Creative Commons Attribution 4.0 International License, which permits use, sharing, adaptation, distribution and reproduction in any medium or format, as long as you give appropriate credit to the original author(s) and the source, provide a link to the Creative Commons license, and indicate if changes were made. The images or other third party material in this article are included in the article's Creative Commons license, unless indicated otherwise in a credit line to the material. If material is not included in the article's Creative Commons license and your intended use is not permitted by statutory regulation or exceeds the permitted use, you will need to obtain permission directly from the copyright holder. To view a copy of this license, visit http://creativecommons. org/licenses/by/4.0/.

(c) The Author(s) 2018 